\title{
Cu-Doped Extremely Small Iron Oxide Nanoparticles with Large Longitudinal Relaxivity: One-Pot Synthesis and in Vivo Targeted Molecular Imaging
}

Irene Fernández-Barahona, ${ }^{\dagger, \bigcirc}$ Lucía Gutiérrez, ${ }^{\S \odot ~ S a b i n o ~ V e i n t e m i l l a s-V e r d a g u e r, ~}{ }^{\Perp}$ Juan Pellico, ${ }^{\dagger,}$

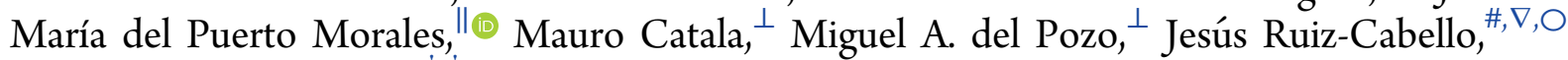
and Fernando Herranz ${ }^{*}, \dagger, \ddagger$

${ }^{\dagger}$ Instituto de Química Médica, CSIC, Juan de la Cierva 3, 28006 Madrid, Spain

${ }^{\ddagger}$ Centro Nacional de Investigaciones Cardiovasculares Carlos III (CNIC) and CIBERES, 28029 Madrid, Spain

${ }^{\S}$ Departamento de Química Analítica, Instituto de Nanociencia de Aragón, Universidad de Zaragoza, Instituto de Ciencia de Materiales de Aragón (ICMA/CSIC) y CIBER-BBN, 50018 Zaragoza, Spain

"Instituto de Ciencia de Materiales de Madrid, CSIC, Sor Juana Inés de la Cruz 3, Cantoblanco, 28049 Madrid, Spain

${ }^{\perp}$ Centro Nacional de Investigaciones Cardiovasculares Carlos III (CNIC), 28029 Madrid, Spain

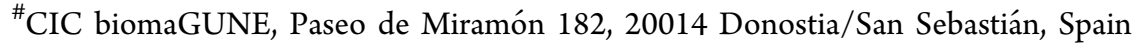

${ }^{\nabla}$ Ikerbasque, Basque Foundation for Science, 48013 Bilbao, Spain

OUniversidad Complutense de Madrid and Centro de Investigación Biomédica en Red de Enfermedades Respiratorias (CIBERES), 28029 Madrid, Spain

Supporting Information

ABSTRACT: Synthesizing iron oxide nanoparticles for positive contrast in magnetic resonance imaging is the most promising approach to bring this nanomaterial back to the clinical field. The success of this approach depends on several aspects: the longitudinal relaxivity values, the complexity of the synthetic protocol, and the reproducibility of the synthesis. Here, we show our latest results on this goal. We have studied the effect of $\mathrm{Cu}$ doping on the physicochemical, magnetic, and relaxometric properties of iron oxide nanoparticles designed to provide positive contrast in magnetic resonance imaging. We have used a one-step, $10 \mathrm{~min}$ synthesis to produce nanoparticles with excellent colloidal stability. We have synthesized three different $\mathrm{Cu}$-doped iron oxide nanoparticles showing modest to very large longitudinal relaxivity values. Finally, we have demonstrated the in vivo use of these kinds of nanoparticles both in angiography and targeted molecular imaging.

\section{INTRODUCTION}

The reduction of transverse relaxation time $\left(T_{2}\right)$ and the longitudinal relaxation time $\left(T_{1}\right)$ of water protons are the two main types of contrasts generated in magnetic resonance imaging (MRI). The first approach produces a darkening of the areas in the image where the probe has accumulated, whereas the second renders a brightening of the image. For technical and physiological reasons, a $T_{1}$-based contrast is preferred by radiologists and physicians for diagnostic purposes. From the imaging point of view, this is a key aspect, being one of the main reasons why "traditional" iron oxide nanoparticles (IONPs), for $T_{2}$-weighted imaging, have not been established as an alternative in the clinical practice. The quest for new iron oxide nanoparticle-based probes for magnetic resonance imaging (MRI) has traditionally focused on the obtainment of transverse relaxivity values $\left(r_{2}\right)$ as large as possible. ${ }^{1}$ Over the last years, the attention has moved to the development of IONPs as "positive" contrast agents, showing large longitudinal relaxivity values $\left(r_{1}\right)$. The rationale behind this approach is trying to get the best of both worlds: commonly used contrast agents in MRI, Gd chelates, and nanoparticles. In other words, to have a good, easy to spot, signal in MRI without a noticeable toxicity, multifunctionalization, and the possibility of easily tuning the pharmacokinetics of the probe. Two aspects are crucial for a good $T_{1}$-based contrast agent: a large $r_{1}$ value and a small $r_{2} / r_{1}$ ratio. The most

Received: October 30, 2018

Accepted: January 22, 2019

Published: February 6, 2019 
(a)

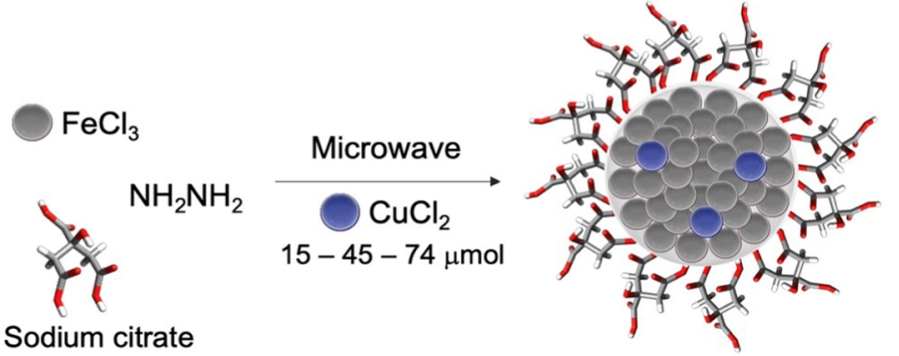

$1.7 \%$ mol doping Cu1.7-NP (b)

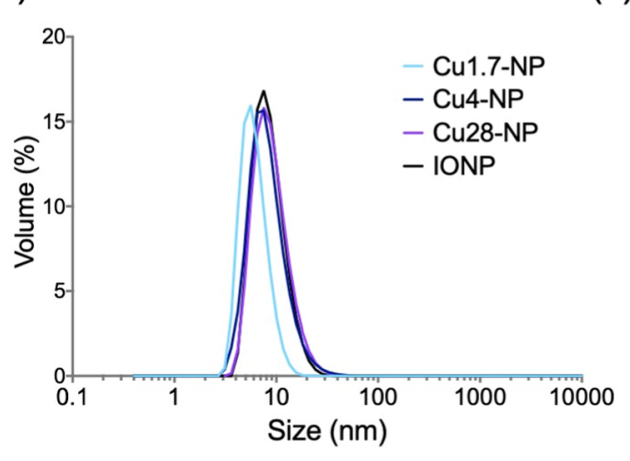

(c)

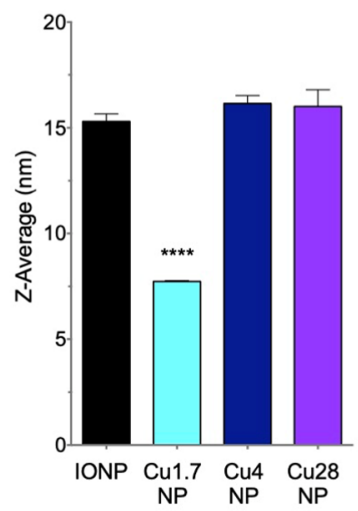

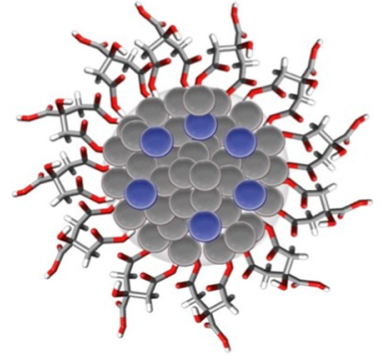

$4 \%$ mol doping Cu4-NP

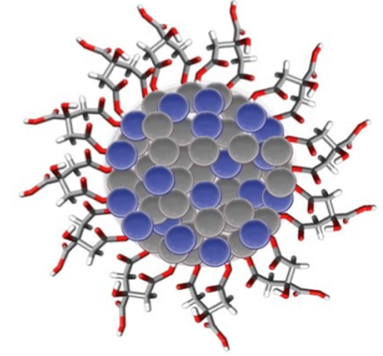

$28 \%$ mol doping

Cu28-NP

(d)

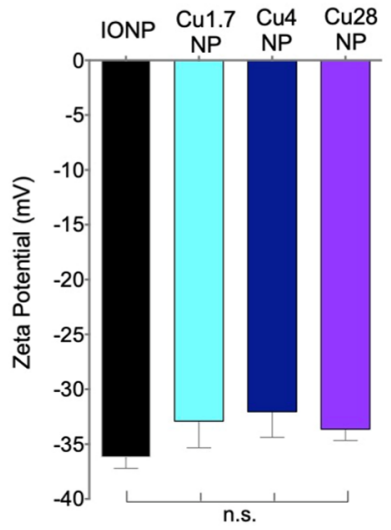

(e)

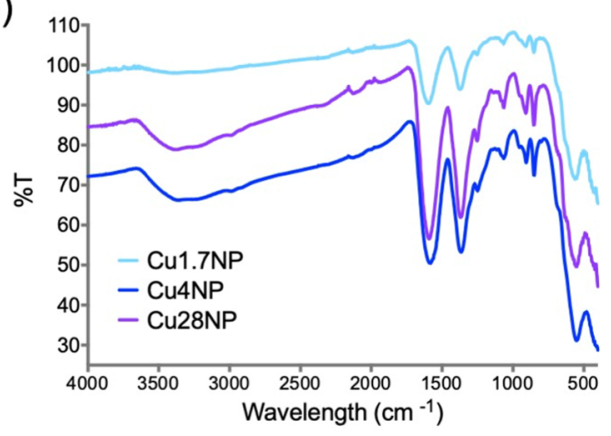

(f)

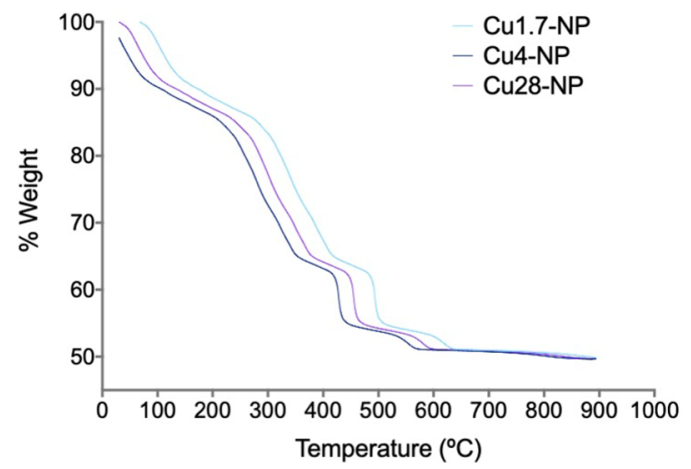

Figure 1. (a) Synthetic scheme for Cu1.7-NP, Cu4-NP, and Cu28-NP; (b) hydrodynamic size distribution, measured by dynamic light scattering (DLS); (c) Z-average size (mean with standard deviation, $N=5, P=0.0001$ ) and (d) $\zeta$-potential for iron oxide nanoparticles (IONPs), Cu1.7-NP, $\mathrm{Cu} 4-\mathrm{NP}$, and Cu28-NP; and (e) Fourier transform infrared (FTIR) spectra and (f) thermogravimetric curves for Cu1.7-NP, Cu4-NP, and Cu28NP.

common approach to obtain IONPs as $T_{1}$ contrast agents is by reducing the core size. Due to the large surface effects, IONPs become more paramagnetic than superparamagnetic and the inner-sphere relaxation mechanism of water protons dominates. ${ }^{2}$ Alternatively, it is possible to modify the chemistry of the coating molecules on the nanoparticles to obtain $T_{1}$ weighted contrast out of IONPs in vivo. ${ }^{3}$ A third option is the core doping of the IONPs with different metals: a few examples describe an increase of the $r_{2}$ values (using $\mathrm{Co}, \mathrm{Ni}$, $\mathrm{Zn}$, or combinations of them $)^{4-7}$ and fewer for positive contrast (mainly with $\mathrm{Mn}$ or Gd as the doping metal). ${ }^{8-10}$ The use of $\mathrm{Cu}$ as a dopant, alone or in combination with other metals, in iron oxide nanoparticles, is limited, compared, for example, to its use as a dopant in $\mathrm{ZnO}$ nanoparticles. $\mathrm{Cu}$ doping effect has been studied in catalysis, ${ }^{11}$ sensor performance, $^{12,13}$ and in the magnetic properties of the nanomaterial. ${ }^{14-16}$ The use of $\mathrm{Cu}$ as a dopant for molecular imaging is limited, with one example, in combination with Co, using nanorods for $\mathrm{MRI}^{17}$ and one example where the ${ }^{64} \mathrm{Cu}$ radioisotope is used for positron emission tomography. ${ }^{18}$ Interestingly, in both cases, the relaxometric properties of the nanomaterials make them suitable exclusively for $T_{2}$-weighted MRI (dark contrast). Furthermore, there are no studies on the use of $\mathrm{Cu}$ doping as a way to obtain nanoparticles for positive contrast MRI.

The use of microwave-driven synthesis (MWS) for the production of nanomaterials is a versatile and highly reproducible approach. We have demonstrated how this methodology can produce, in extremely short times, homogeneous nanoparticles with excellent properties for molecular imaging. ${ }^{3,19}$ However, in the production of nanoparticles for $T_{1}$-weighted MRI, the use of MWS is far from common. Most of the syntheses used for the production of $T_{1}$ nanoparticles are characterized by time-consuming protocols, $^{8-10,20,21}$ multistep approaches, ${ }^{9,10,22-26}$ or the use of organic solvents. ${ }^{22-26}$

Here, we have studied the effect of $\mathrm{Cu}$ doping of iron oxide nanoparticles on their relaxometric properties. In a $10 \mathrm{~min}$, 

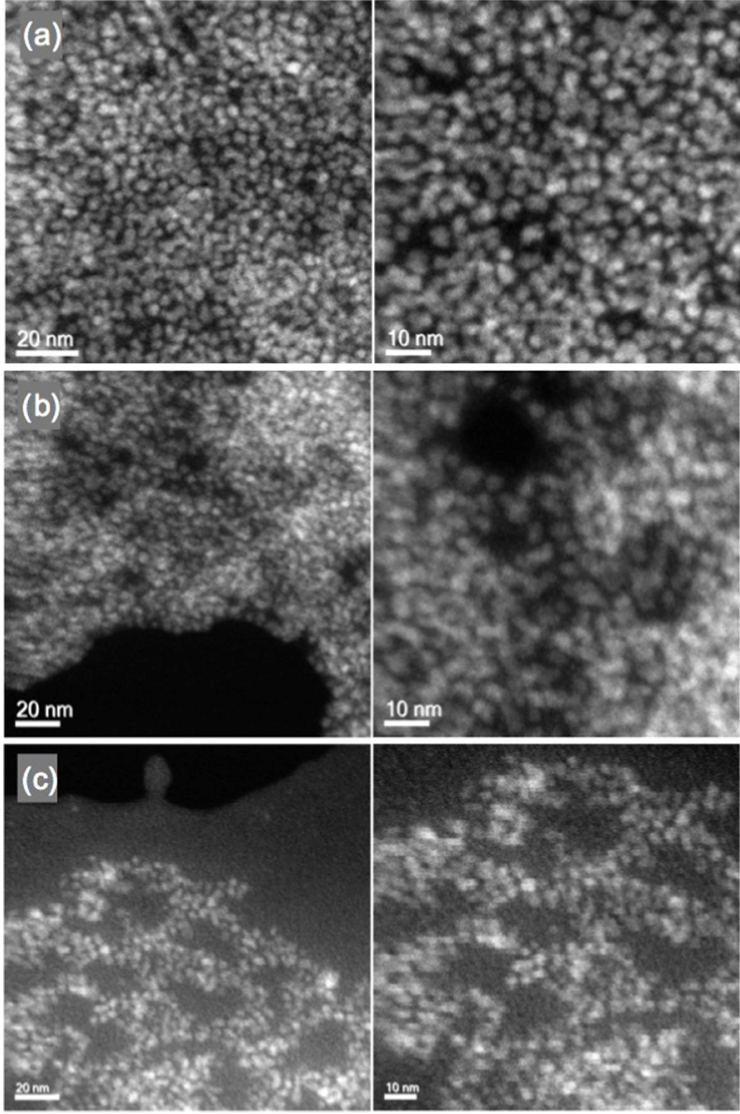

d)

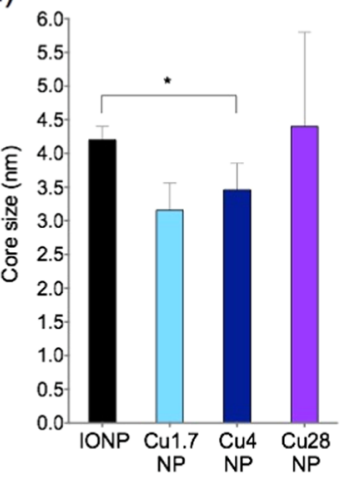

(e)

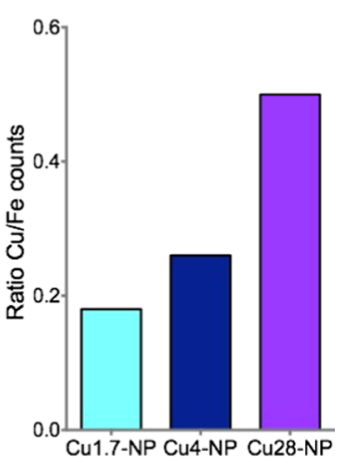

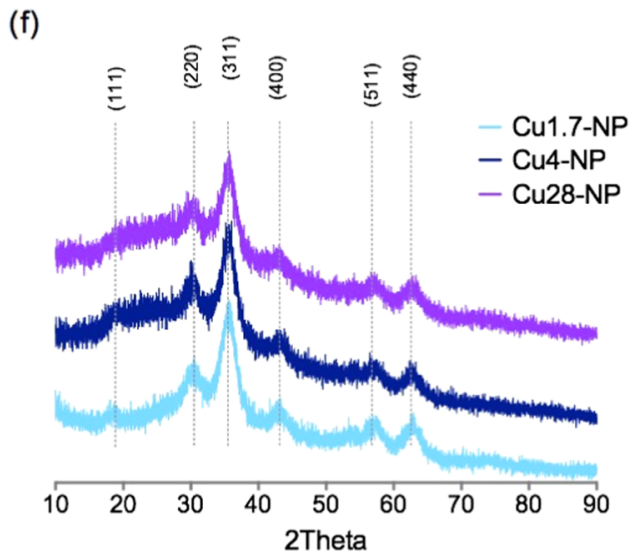

Figure 2. Selected STEM-HAADF images of (a) Cu1.7-NP, (b) Cu4-NP, and (c) Cu28-NP; (d) core size measured by STEM (mean with standard deviation, $N=30, P=0.01$ ); (e) ratio of $\mathrm{Cu} / \mathrm{Fe}$ counts in $\mathrm{EDX}$; and (f) XRD profiles for the three samples.

one-pot synthesis, we have produced $\mathrm{Cu}$ core-doped IONP ( $\mathrm{Cu}-\mathrm{NP})$ with the largest longitudinal relaxivity value described so far, at clinically relevant magnetic fields. We have fully characterized the new nanomaterial and demonstrated its suitability for in vivo use in angiography and targeted molecular imaging for the enhanced diagnosis of tumors in animal models.

\section{RESULTS AND DISCUSSION}

2.1. One-Pot Microwave-Driven Synthesis of $\mathrm{Cu}$ Doped Hydrophilic Iron Oxide Nanoparticles. Microwave synthesis of nanoparticles is a reliable, reproducible, and extremely fast way for the production of small iron oxide nanoparticles (IONPs). We recently used this approach to tune the relaxivity values of extremely small iron oxide nanoparticles, from $T_{2}$ agents to $T_{1}$ agents. ${ }^{3}$ Here, we have synthesized three different iron oxide nanoparticles with an increasing amount of $\mathrm{Cu}$ core doping (Figure 1a). In a typical synthesis, we mixed $\mathrm{FeCl}_{3}$, sodium citrate, and hydrazine with different amounts of $\mathrm{CuCl}_{2}(15,45$, and $74 \mu \mathrm{mol})$; after 10 min of heating at $120^{\circ} \mathrm{C}$, we purified the nanoparticles by size exclusion chromatography. After purification, we quantified the amount of $\mathrm{Cu}$ in the samples by inductively coupled plasma mass spectrometry (ICP-MS): $1.7 \mathrm{~mol} \%$ (sample Cu1.7-NP), $4 \mathrm{~mol} \%$ (sample Cu4-NP), and $28 \mathrm{~mol} \%$ (sample Cu28-NP).

2.2. Physicochemical Characterization of $\mathrm{Cu}$-Doped Iron Oxide Nanoparticles. Hydrodynamic size distributions (Figure $1 b$ ) are narrow for all of the samples. Samples IONP, Cu4-NP, and Cu28-NP show the same size distribution, whereas for the sample with the lowest amount of $\mathrm{Cu}(\mathrm{Cu} 1.7$ $\mathrm{NP}$ ), the size is smaller. This is confirmed by plotting the $Z$ average values for each sample (Figure 1c). Sample Cu1.7-NP shows a much smaller size $(7.7 \pm 0.6 \mathrm{~nm})$ than the other nanoparticles in this study; values for IONP, Cu4-NP, and $\mathrm{Cu} 28-\mathrm{NP}$ are $15.0 \pm 1.0,16.1 \pm 0.7$, and $16.0 \pm 1.5 \mathrm{~nm}$, respectively. $\zeta$-Potential measurements show no significant differences between samples (Figure 1d) with homogeneous values around $-34 \mathrm{mV}$ as expected for citrate-coated nanoparticles.

FTIR spectra for the three $\mathrm{Cu}$-doped samples show the expected bands (Figure 1e); bands corresponding to citrate coating appear at 1580,1380, and $1090 \mathrm{~cm}^{-1}$. The band with maxima at $1580 \mathrm{~cm}^{-1}$ is wider for Cu28-NP (from 1460 to $1720 \mathrm{~cm}^{-1}$ ), most likely due to the presence of the $\mathrm{Cu}-\mathrm{O}$ band that normally appears at around $1640 \mathrm{~cm}^{-1}$. A strong band due to iron oxide is observed in all three spectra at 560 $\mathrm{cm}^{-1}$. Further analysis in the region between 800 and $400 \mathrm{~cm}^{-1}$ shows at least a band at a higher wavelength than $600 \mathrm{~cm}^{-1}$, indicating that the sample is mainly composed of maghemite rather than magnetite. ${ }^{3}$ Thermogravimetric analysis shows the same curves for the three samples. We found three different steps at around 120,380 , and $480{ }^{\circ} \mathrm{C}$; this mass loss in a stepwise manner is typical of citrate-coated nanoparticles. ${ }^{27}$ All samples show a thick organic layer, about $50 \%$ of weight loss, which ensures their colloidal stability and the availability of carboxylic groups for bioconjugation.

Electron microscopy of these samples is often difficult; a small core and a thick organic layer complicate acquiring quality images. The same happens with X-ray diffraction 
(XRD), which normally produces broad peaks difficult to identify. To ameliorate this issue, we treated the samples (Cu1.7-NP, Cu4-NP, and Cu28-NP), prior analysis, with the enzyme citrate lyase. This enzyme catalyzes the reversible aldol cleavage of citrate to oxaloacetate and acetate. Our hypothesis was that by cleaving citrate the organic layer would be at least partially reduced so scanning transmission electron microscopy (STEM) images would be better and XRD peaks would be more clearly defined. Figure 2 shows selected STEM-HAADF images of the three samples (more images are shown in the Supporting Information). Images reveal small cores for all of them, similar to IONPs produced with this approach. ${ }^{3}$ We can observe individual, dispersed nanoparticles, for all three samples. The core size of the nanoparticles, measured by STEM, also shows that Cu1.7-NP has the lowest value (3.2 \pm $0.9 \mathrm{~nm})$ and Cu4-NP has a similar core size $(3.5 \pm 0.8 \mathrm{~nm})$, whereas IONP $(4.2 \pm 1.0 \mathrm{~nm})$ and Cu28-NP $(4.4 \pm 2.9 \mathrm{~nm})$ show the largest values. Interestingly, Cu28-NP, the sample with the largest amount of $\mathrm{Cu}$, shows a much higher heterogeneity than the other nanoparticles in the core size (Figure 2d), which is also reflected in the quality of the images (Figure 2c). Energy dispersive X-ray (EDX) analysis of the samples confirms the results obtained by ICP-MS, an increase in the $\mathrm{Cu} / \mathrm{Fe}$ ratio from $\mathrm{Cu} 1.7-\mathrm{NP}$ to $\mathrm{Cu} 28$-NP (Figure 2e).

The effect of the enzyme is noticeable in the quality of the XRD spectra. A comparison with previous data on IONP, without enzymatic treatment, clearly shows that now the peaks are more defined and easier to identify. ${ }^{3}$ The increase in $\mathrm{Cu}$ doping results in a slight displacement of the main peak (311) toward larger angles and the decrease of the intensity in the peaks. ${ }^{15}$ Using the Scherrer equation, we calculated the crystallite sizes for the three nanoparticles: $2.9 \mathrm{~nm}$ for Cu1.7-NP; $3.5 \mathrm{~nm}$ for Cu4-NP; and $3.4 \mathrm{~nm}$ for $\mathrm{Cu} 28-\mathrm{NP}$, in good agreement with the core sizes measured by STEM. The field-dependent magnetization data (Figure 3a) show negligible coercivity values for all of the samples, confirming their superparamagnetic behavior. The incorporation of increasing amounts of $\mathrm{Cu}$ significantly reduces the saturation magnetization of the sample, even for the smallest amounts of $\mathrm{Cu}$.

Saturation magnetization values are $76.6 \pm 0.3 \mathrm{emu} \mathrm{g}^{-1} \mathrm{Fe}+$ $\mathrm{Cu}$ for $\mathrm{Cu} 1.7-\mathrm{NP}, 56.2 \pm 0.3 \mathrm{emu}^{-1} \mathrm{Fe}+\mathrm{Cu}$ for $\mathrm{Cu} 4-\mathrm{NP}$, and $43.8 \pm 0.4 \mathrm{emu} \mathrm{g}^{-1} \mathrm{Fe}+\mathrm{Cu}$ for $\mathrm{Cu} 28-\mathrm{NP}$, showing the dilution effect given by the increasing proportion of copper. However, if we express the saturation magnetization on iron basis, we obtain $78.2 \pm 0.3 \mathrm{emu} \mathrm{g}^{-1} \mathrm{Fe}$ for Cu1.7-NP, $58.4 \pm$ $0.3 \mathrm{emu} \mathrm{g}^{-1} \mathrm{Fe}$ for $\mathrm{Cu} 4-\mathrm{NP}$, and $63.3 \pm 0.4 \mathrm{emu} \mathrm{g}^{-1} \mathrm{Fe}$ for Cu28-NP. This unexpected high value for Cu28-NP supports the core-shell structure for this sample where the excess of $\mathrm{Cu}$ is mainly located on the surface of the nanoparticle core. The temperature dependence of the out-of-phase susceptibility shows maxima located in the range between 15 and $22 \mathrm{~K}$ for all samples, together with maxima in the in-phase susceptibility at slightly higher temperatures (data not shown) typical of the relaxation procedure of nanoparticles (Figure $3 \mathrm{~b}$ ). The location of the out-of-phase susceptibility is influenced by the size distribution, composition, and aggregation (dipolar interactions) of the particles. A mixture of factors affects our results; the increase in the $\mathrm{Cu}$ amount (that would probably reduce the saturation magnetization values and the temperature location of the susceptibility maxima) is associated with an increase in the particle size (Figures 1 and 2) that would have the opposite effect. The negligible out-of-phase susceptibility values at temperatures above $100 \mathrm{~K}$ indicate (a)
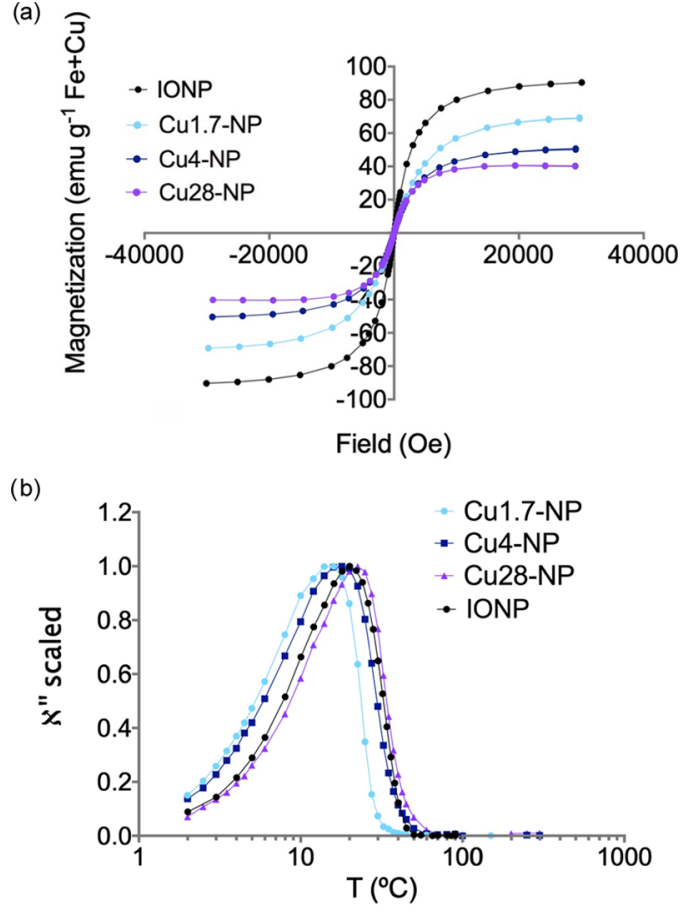

Figure 3. (a) Magnetization curves at $298 \mathrm{~K}$ and (b) temperature dependence of AC magnetic susceptibility for IONP, Cu1.7-NP, Cu4$\mathrm{NP}$, and Cu28-NP.

the superparamagnetic behavior of these particles at room temperature, in agreement with the negligible coercivity observed in the field-dependent magnetization data.

2.3. Relaxometric Characterization of $\mathrm{Cu}$-Doped Nanoparticles. After this comprehensive physicochemical characterization, we focused on our main goal, that is, the study of the effect on the relaxometric values of $\mathrm{Cu}$ core doping. Figure $4 a-c$ shows the plot of averaged points for $r_{2}$ and $r_{1}$ for the three nanoparticles. In all cases, we observe a good linear fitting of the measured points and a good reproducibility. A comparison of the relaxometric values for the three $\mathrm{Cu}$-doped nanoparticles (Figure $4 \mathrm{~d}-\mathrm{f}$ ) shows some remarkable results. The longitudinal relaxivity $\left(r_{1}\right.$, Figure $\left.4 \mathrm{~d}\right)$ shows that sample Cu4-NP has a large $r_{1}$ value $(15.7 \pm 0.6$ $\left.\mathrm{mM}^{-1} \mathrm{~s}^{-1}\right)$, significantly larger than that for iron oxide nanoparticles $\left(11.9 \pm 0.3 \mathrm{mM}^{-1} \mathrm{~s}^{-1}\right)$. The $r_{2}$ value is also larger for Cu4-NP; however, the $r_{2} / r_{1}$ ratio is pretty low (2.1), in summary, excellent conditions for $T_{1}$-weighted magnetic resonance imaging. Sample $\mathrm{Cu} 1.7-\mathrm{NP}$ also shows an improved performance in the longitudinal relaxivity $\left(13.6 \pm 0.3 \mathrm{mM}^{-1}\right.$ $\mathrm{s}^{-1}$ ), compared to that of IONP. The transversal relaxivity value is similar to that for $\mathrm{Cu} 4-\mathrm{NP}$, and the $r_{2} / r_{1}$ ratio is slightly larger. These properties make Cu1.7-NP also an optimal contrast agent for $T_{1}$-weighted imaging, second only to the excellent results for $\mathrm{Cu} 4-\mathrm{NP}$. Finally, the sample with the largest amount of $\mathrm{Cu}$ shows poor performance as an MRI contrast agent. The $r_{1}$ value $\left(8.5 \pm 1.3 \mathrm{mM}^{-1} \mathrm{~s}^{-1}\right)$ is much smaller than the value for IONP, and the $r_{2} / r_{1}$ ratio is the largest for the series (2.4). The relaxometric study shows that, under tested conditions, a $4 \mathrm{~mol} \%$ doping provides the best properties for an improved $T_{1}$ contrast. A large doping percentage, as in $\mathrm{Cu} 28-\mathrm{NP}$, renders nanoparticles with worse $T_{1}$ contrast than that of nanoparticles without doping. 
(a)

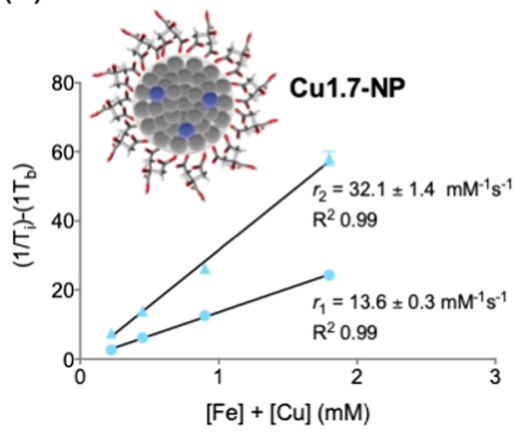

(d)

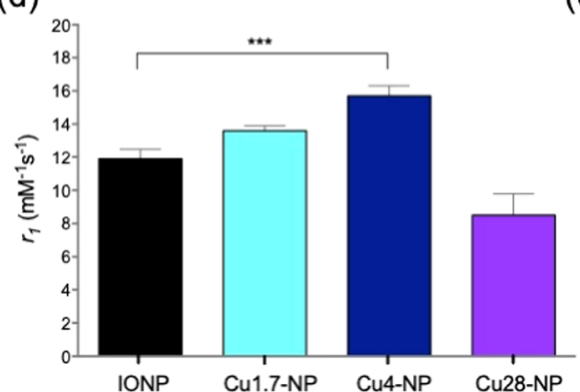

(b)

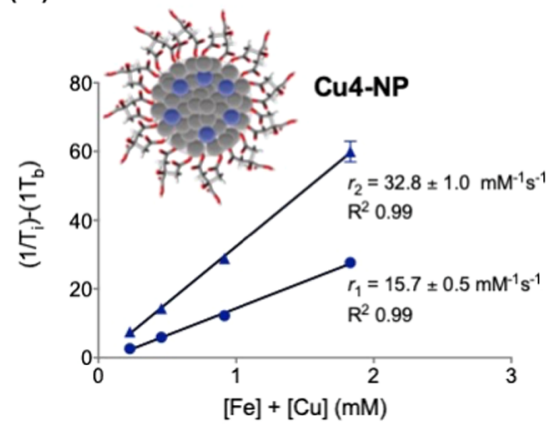

(e)

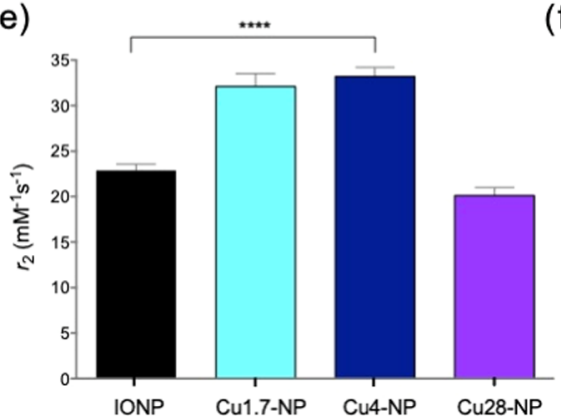

(c)

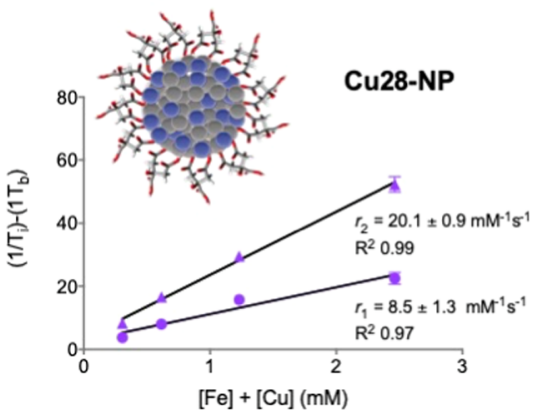

(f)

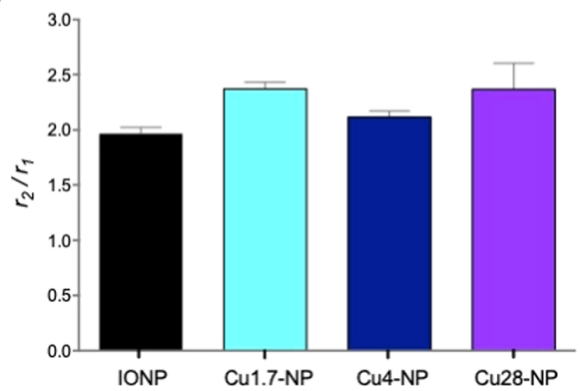

Figure 4. Plots of relaxation rates, $T_{1}$ and $T_{2}$, against iron and copper concentration for (a) Cu1.7-NP, (b) Cu4-NP, and (c) Cu28-NP. Plots of (d) $r_{1}$ (mean with standard deviation, $N=5, P=0.001$ ), (e) $r_{2}$ (mean with standard deviation, $N=5, P=0.0001$ ), and (f) $r_{2} / r_{1}$ for IONP, Cu1.7-NP, Cu4-NP, and Cu28-NP.

Table 1. Relaxometric Properties of Nanoparticles with Large $r_{1}$ Values $\left(>4 \mathrm{mM}^{-1} \mathrm{~s}^{-1}\right)$

\begin{tabular}{|c|c|c|c|c|c|c|c|}
\hline name & composition & $r_{1}\left(\mathrm{mM}^{-1} \mathrm{~s}^{-1}\right) /$ field $(\mathrm{T})$ & $r_{2}\left(\mathrm{mM}^{-1} \mathrm{~s}^{-1}\right) /$ field $(\mathrm{T})$ & $r_{2} / r_{1}$ & $\begin{array}{l}\text { core size } \\
(\mathrm{nm})\end{array}$ & $\begin{array}{c}H_{\mathrm{D}} \\
(\mathrm{nm})\end{array}$ & references \\
\hline $\mathrm{MnIO}$ & diethylene glycol-BSA-Mn-iron oxide & $8.24 / 0.55$ & $17.95 / 0.55$ & 2.2 & 5.0 & 5.9 & 9 \\
\hline $\mathrm{DIO}-\mathrm{Cu}$ & dextran-iron oxide- $\mathrm{Cu}$ & $17.1 / 1.4$ & $135.8 / 1.4$ & 7.9 & 5.3 & 45.7 & 18 \\
\hline $\mathrm{MnFe}_{2} \mathrm{O}_{4}$ & polymers-Mn-iron oxide & $6.61 / 4.7$ & $35.92 / 4.7$ & 5.4 & 2.2 & 5.6 & 8 \\
\hline MION & dextran-iron oxide & $16.5 / 0.47$ & $34.8 / 0.47$ & 2.1 & 4.6 & 20 & 28 \\
\hline $\mathrm{PO}-\mathrm{PEG}-\mathrm{IO}$ & phosphine oxide-PEG-iron oxide & $4.78 / 3$ & $17.5 / 3$ & 3.7 & 2.2 & 15 & 25 \\
\hline $\mathrm{Fe}_{2} \mathrm{O}_{3}$-citrate & citric acid-iron oxide & $14.5 / 1.4$ & $66.9 / 1.4$ & 4.6 & 4.8 & 18 & 24 \\
\hline $\mathrm{PEG}_{1100}-\mathrm{IO}$ & $\mathrm{PEG}_{1100}$-iron oxide & $13 / 1.4$ & $42 / 1.4$ & 3.2 & 2.8 & 10 & 22 \\
\hline $\mathrm{Fe}_{3} \mathrm{O}_{4} @ \mathrm{SiO}_{2}(\mathrm{Gd}-\mathrm{DTPA})$ & silica-iron oxide-Gd & $4.2 / 3$ & $17.4 / 3$ & 4.1 & 27 & & 29 \\
\hline UMIONS & polymers-iron oxide & $8.3 / 4.7$ & $35.1 / 4.7$ & 4.2 & 3.3 & 7.5 & 20 \\
\hline SPIONS & carboxyPEG-iron oxide & $19.7 / 1.5$ & $39.5 / 1.5$ & 2.0 & 5.4 & 10 & 21 \\
\hline IONP & citric acid-iron oxide & $11.9 / 1.5$ & $22.9 / 1.5$ & 1.9 & 4.2 & 15.0 & 3 \\
\hline Cu4-NP & citric acid-Cu-doped iron oxide & $15.7 / 1.5$ & $32.8 / 1.5$ & 2.1 & 3.5 & 16.1 & this work \\
\hline
\end{tabular}

Magnetic measurements suggest a core-shell structure for $\mathrm{Cu} 28-\mathrm{NP}$, with most of the $\mathrm{Cu}$ atoms on the surface; this explains the small $r_{1}$ value since $\mathrm{Cu}$ atoms on the surface would reduce the contribution of the inner-sphere relaxation mechanism. In Cu1.7-NP, we see an improvement in the $T_{1}$ signal but lower than for the $4 \%$ doping conditions. It seems that around this doping percentage we obtained the appropriate reduction of the magnetization, keeping enough $\mathrm{Fe}$ atoms on the surface. The $1.7 \%$ doping is too small to see the full effect, whereas the $28 \%$ doping seems too large to keep the desirable relaxometric properties of the iron oxides prepared this way. Surface coating thickness is a key parameter to improve $r_{1}$ values, as we have demonstrated before. ${ }^{3} \mathrm{Cu} 4$ NP coating is thinner than IONP coating; this feature also contributes to increase the longitudinal relaxivity value (Figure S4). The $r_{1}$ values shown by $\mathrm{Cu} 4-\mathrm{NP}$ are similar to or larger than those of many clinical Gd- and Mn-based compounds, and $\mathrm{Cu} 4-\mathrm{NP}$ has a low $r_{2} / r_{1}$ ratio. $^{30} \mathrm{We}$ can compare these results with those of nanoparticles for $T_{1}$-weighted MRI published in recent years (Table 1). This table includes not only iron oxide nanoparticles but also Gd- and Mn-based compounds. The performance of an MRI contrast agent for $T_{1^{-}}$ weighted imaging is based on the $r_{1}$ value and the $r_{2} / r_{1}$ ratio. The larger the $r_{1}$ value and the smaller the $r_{2} / r_{1}$ ratio, the better. Considering this, Cu4-NPs are one of the best suited nanoparticles for positive contrast MRI thus far reported. These nanoparticles have one of the largest $r_{1}$ values and one of the smallest $r_{2} / r_{1}$ ratios. It is noteworthy how the combination of citric acid and iron oxide can produce nanoparticles with large $r_{1}$ values but poor $r_{2} / r_{1}$ ratios. $^{24}$ One of the few examples of iron oxide nanoparticles doped with $\mathrm{Cu}$ shows good relaxometric properties but for $T_{2^{-}}$ weighted imaging, confirming the nanoparticles must be considered as a whole to optimize the MRI performance. SPIONS $^{21}$ and $\mathrm{MION}^{28}$ show remarkable properties as $T_{1}$ agents, with large $r_{1}$ values. However, this is usually achieved 


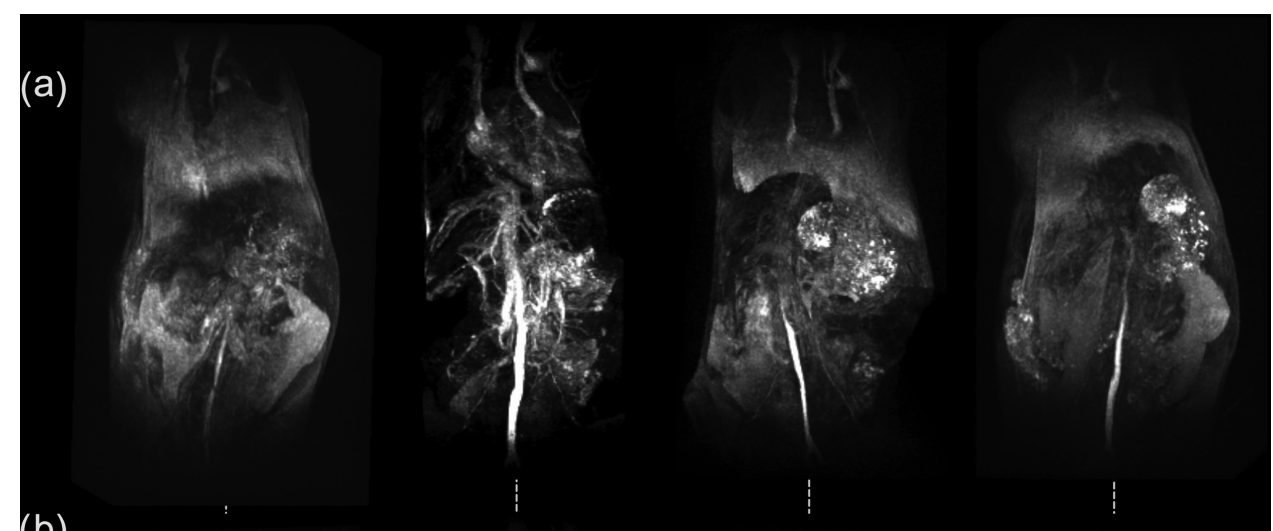

(b)

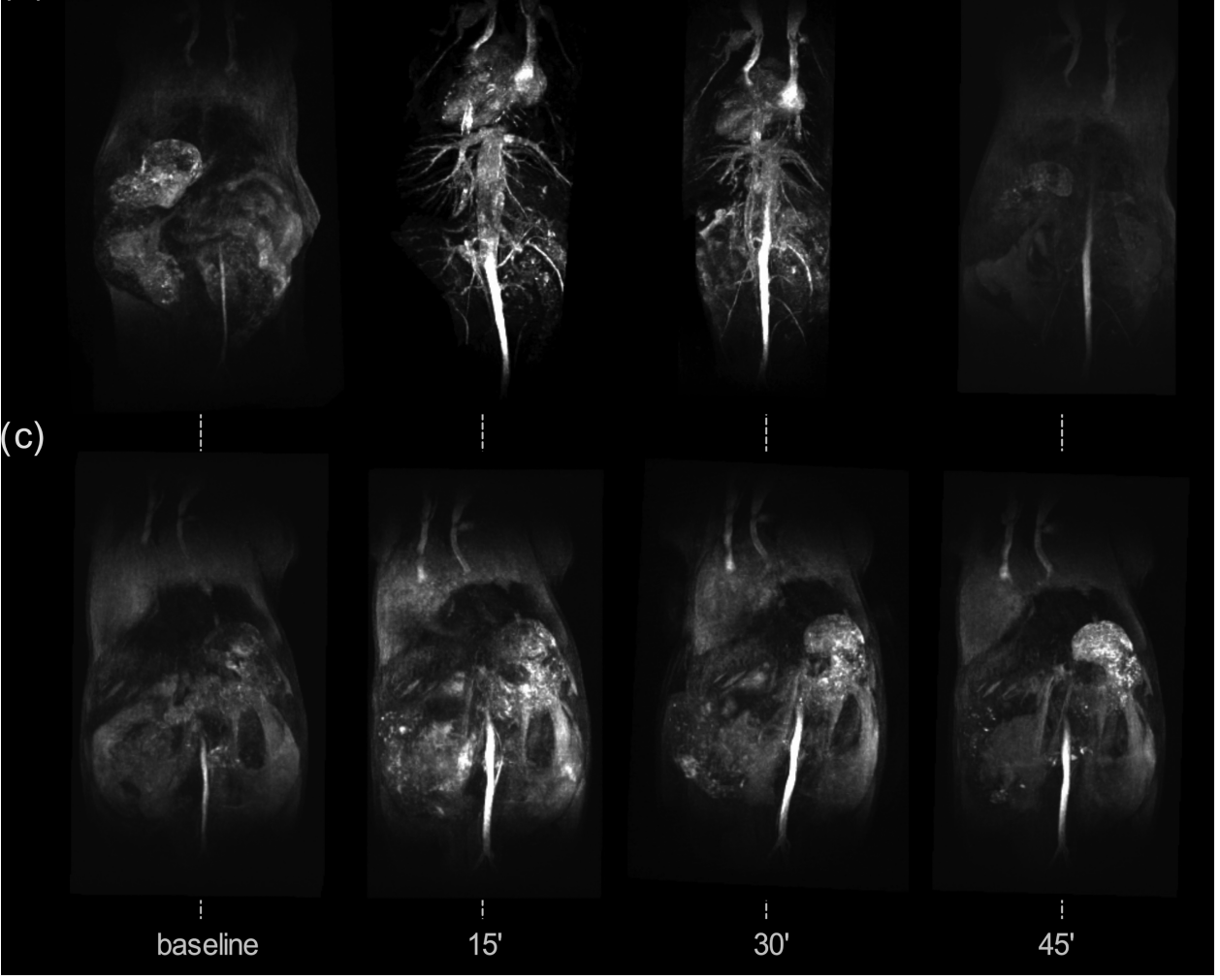

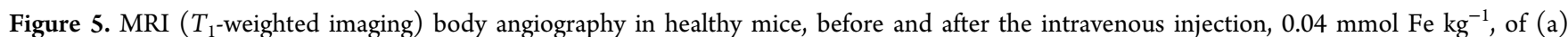
Cu1.7-NP, (b) Cu4-NP, and (c) Cu28-NP.

after a lengthy and time-consuming synthesis and purification. This can compromise the reproducibility and is certainly not the best scenario for clinical translation. These nanoparticles also show a relatively thin organic layer that could complicate their stability under physiological conditions. In the case of $\mathrm{Cu} 4-\mathrm{NP}$, we achieved one of the best relaxometric properties, in one single step and with a thick organic coating, ensuring their colloidal stability under most relevant physiological conditions.

2.4. Magnetic Resonance Angiography with $\mathrm{Cu}-$ Doped Nanoparticles. A good contrast agent for in vivo magnetic resonance imaging must have excellent relaxometric properties. This alone is insufficient, and appropriate in vivo behavior, factors like pharmacokinetics and colloidal stability under physiological conditions, must be checked; for this, the best test is an in vivo image. We carried out magnetic resonance angiography with $\mathrm{Cu} 1.7-\mathrm{NP}, \mathrm{Cu} 4-\mathrm{NP}$, and $\mathrm{Cu} 28$ NP (Figure 5) to check the potential of their $T_{1}$ signals. We acquired images before nanoparticle injection (baseline) and
15,30 , and 45 min post injection. The differences between the nanoparticle samples are clear. Angiography with Cu4-NP provides high-quality images with fine details of the vasculature up to $30 \mathrm{~min}$ post injection.

The use of Cu1.7-NP provides positive contrast after 15 min, although the signal is not that clear as that for $\mathrm{Cu} 4-\mathrm{NP}$; then, the signal becomes weaker after $30 \mathrm{~min}$ of circulation. We attribute this difference to the $r_{1}$ and $r_{2} / r_{1}$ values for $\mathrm{Cu} 4$ $\mathrm{NP}$ since the circulating time for Cu1.7-NP is similar to that for Cu4-NP (Figure S5), discarding a rapid renal elimination as the cause for this difference. Angiographic images with $\mathrm{Cu} 28$ NP show the poorest results due to the smaller $r_{1}$ value, half of that for $\mathrm{Cu} 4-\mathrm{NP}$, and a shorter circulation time.

\subsection{Tumor Detection by Magnetic Resonance} Imaging: Comparison between IONP and Cu4-NP. We have shown that $\mathrm{Cu} 4-\mathrm{NPs}$ provide an excellent signal in angiographic MRI with adequate in vivo behavior. We wanted to verify the use of this new contrast agent using actively targeted molecular imaging and compare it with the undoped 


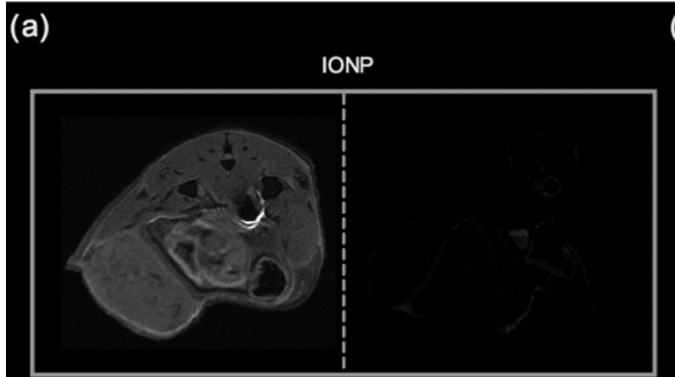

(b)
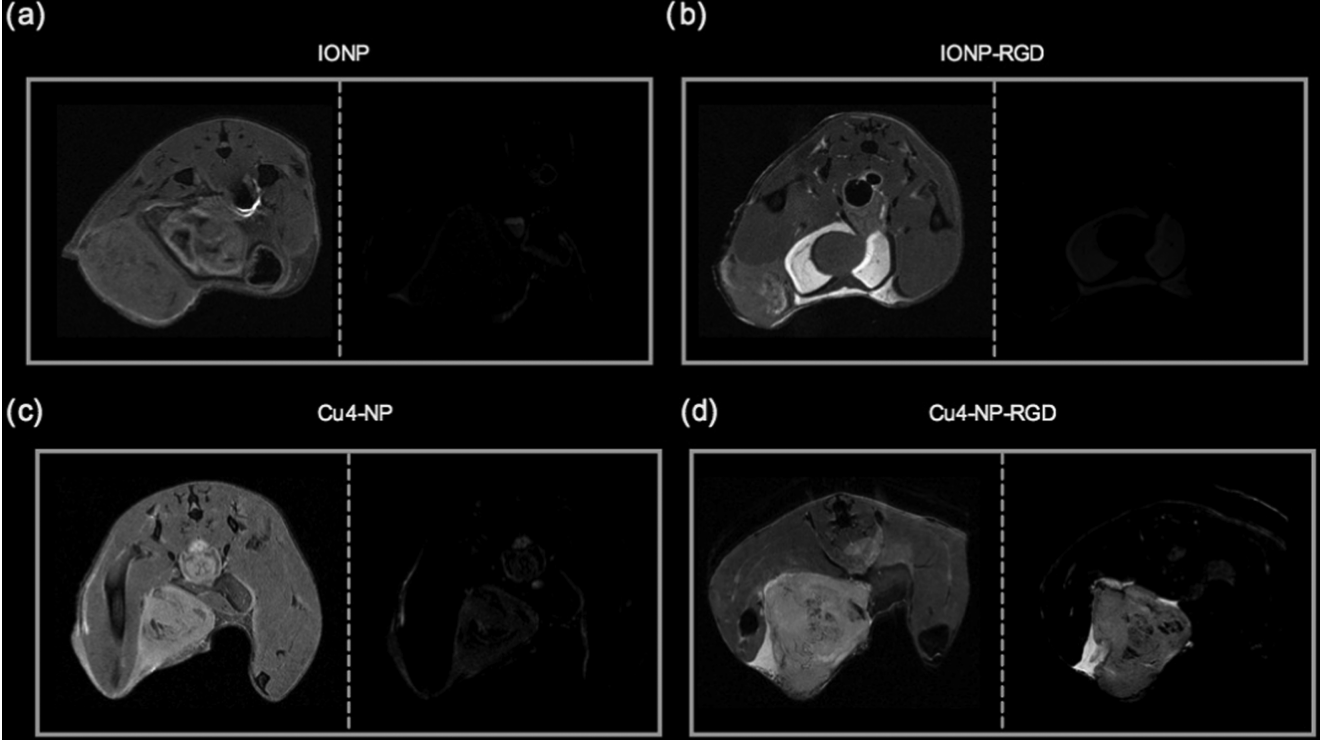

(d)

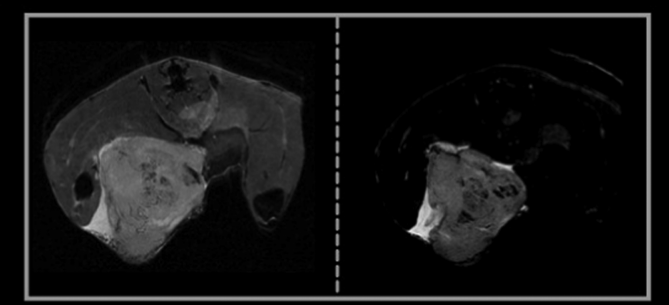

Figure 6. Magnetic resonance imaging of breast tumor-bearing mice allografts $1 \mathrm{~h}$ after the intravenous injection of $0.05 \mathrm{mmol} \mathrm{Fe} \mathrm{kg}^{-1}$ of $(\mathrm{a})$ IONP, (b) IONP-RGD, (c) Cu4-NP, and (d) Cu4-NP-RGD. Left panels show normal contrast images, and right panels are obtained after setting the muscle intensity to 0 to highlight the possible increase in the tumor signal. All MRI images were acquired (see the Experimental Section) with the same parameters.

nanoparticles, IONPs. We designed a classical experiment, that is, targeting vascular angiogenesis toward integrin $\alpha_{\mathrm{v}} \beta_{3}$ in tumor models using RGD peptide as a vector. We conjugated the peptide on the surface of both IONP and Cu4-NP, which are similar in terms of chemical composition and thickness of the organic layer. Using EDC/sulfoNHS to activate carboxylic groups on the surface, we bound the RGD peptide by amide formation with citric acid carboxylate groups. We measured the hydrodynamic size and $\zeta$-potential for the RGD-functionalized nanoparticles (Figure S6), showing a different value of $31.9 \mathrm{~nm}$ for $\mathrm{Cu} 4-\mathrm{NP}-\mathrm{RGD}$ and $37.3 \mathrm{~nm}$ for IONP-RGD. $\zeta$-Potential values are the same in both cases, suggesting a similar functionalization of the surface $(-12.9 \mathrm{mV}$ for Cu4-NP-RGD vs $-12.0 \mathrm{mV}$ for IONP-RGD). After RGD quantification on the nanoparticle surface (Bradford assay), we obtained a value of 16.1 RGD molecules per IONP and 15.9 molecules of RGD per Cu4-NP. We performed in vivo MRI experiments in mouse breast cancer allograft models, after the intravenous injection of four different nanoparticles, IONP, Cu4-NP, IONP-RGD, and $\mathrm{Cu} 4-\mathrm{NP}-\mathrm{RGD}$.

Figure 6 shows the MR data obtained $1 \mathrm{~h}$ after the intravenous injection of the different NPs. We show images with the normal contrast (left panels in each image) and also setting the muscle contrast intensity to 0 (right panels in each image) to better appreciate the possible increase in the tumor signal. Differences are dramatic. First, we can discard the accumulation of the nanoparticles in the tumor due to the enhanced permeability and retention effect since there is no significant signal in the tumor after the injection of either IONP (Figure 6a) or Cu4-NP (Figure 6c). The situation is different after the injection of the functionalized NPs. The image after the injection of IONP-RGD shows a modest increase in the tumor signal (Figure S7); however, when muscle pixels are set to 0 , the tumor is barely distinguishable from this reference. The situation is completely different for $\mathrm{Cu} 4-\mathrm{NP}-\mathrm{RGD}$. Figure 6d (left panel) clearly shows an increase in the signal compared to that from the muscle and also when compared to that in Figure $6 c$. This signal increase is even more clearly displayed when the muscle intensity is set to 0 ; the tumor is clearly observable owing to a $30 \%$ increase in the MRI signal (Figure S6). The difference in hydrodynamic size between IONP-RGD and Cu4-NP-RGD (Figure S6) can contribute to this difference but in a minor proportion. The main reason for this outstanding result obtained with Cu4-NP-RGD is the very large $r_{1}$ value obtained, significantly larger than the $r_{1}$ value for the undoped nanoparticles. To further explain this, we measured the relaxivity values for $\mathrm{Cu} 4-$ $\mathrm{NP}$ and IONP at $7 \mathrm{~T}$. We measured $r_{1}$ values of $2.7 \mathrm{mM}^{-1} \mathrm{~s}^{-1}$ for Cu4-NP and $2.8 \mathrm{mM}^{-1} \mathrm{~s}^{-1}$ for IONP. However, the $r_{2}$ values are $38.7 \mathrm{mM}^{-1} \mathrm{~s}^{-1}$ for $\mathrm{Cu} 4-\mathrm{NP}$ and $88.5 \mathrm{mM}^{-1} \mathrm{~s}^{-1}$ for IONP. These values permit us to get a good $T_{1}$ contrast at a high field with Cu4-NP samples but not with IONP because of the large $r_{2}$ value, explaining the large differences observed at 7 $\mathrm{T}$ in vivo for tumor detection.

\section{CONCLUSIONS}

The search for better contrast agents for magnetic resonance imaging has implications for both basic and translational research. The synthesis and application of nanoparticles for positive contrast are particularly interesting since these will boost the use of nanomaterials in this imaging technique, key for many clinical applications. In this work, we show for the first time how the use of $\mathrm{Cu}$, as a dopant, enhances the positive contrast in vivo performance of iron oxide nanoparticles. We have fully characterized the nanoparticles and demonstrated the in vivo use of this nanomaterial, both as a blood pool contrast agent and for targeted molecular imaging, which is key for further development and applications. The relaxometric values, the type of synthesis, and the in vivo performance make $\mathrm{Cu} 4-\mathrm{NP}$ a remarkable candidate for future clinical translation.

\section{EXPERIMENTAL SECTION}

4.1. Synthesis of IONP-Citrate Samples. A mixture of $\mathrm{FeCl}_{3} \cdot 6 \mathrm{H}_{2} \mathrm{O}(75 \mathrm{mg})$ and citric acid trisodium salt $(80 \mathrm{mg})$ was dissolved in water $(9 \mathrm{~mL})$. Subsequently, hydrazine 
monohydrate $(1 \mathrm{~mL})$ was added and the mixture was rapidly introduced into the microwave. Samples were heated under vigorous stirring at $240 \mathrm{~W}$ for $10 \mathrm{~min}$ at $120^{\circ} \mathrm{C}$. Once this step was completed, nanoparticles were purified through a gel filtration column (PD10) to eliminate unreacted species and stored in glass vials for further characterization.

4.2. Synthesis of Cu-NP-Citrate Samples. A mixture of $\mathrm{FeCl}_{3} \cdot 6 \mathrm{H}_{2} \mathrm{O}(75 \mathrm{mg})$, citric acid trisodium salt $(80 \mathrm{mg})$, and $\mathrm{CuCl}_{2}(2,6$, or $10 \mathrm{mg})$ was dissolved in water $(9 \mathrm{~mL})$. Subsequently, hydrazine monohydrate $(1 \mathrm{~mL})$ was added and the mixture was rapidly introduced into the microwave. Samples were heated under vigorous stirring at $240 \mathrm{~W}$ for 10 $\min$ at $120{ }^{\circ} \mathrm{C}$. Once this step was completed, nanoparticles were purified through a gel filtration column (PD10) to eliminate unreacted species and stored in glass vials for further characterization.

4.3. Cu-NP Treatment with Citrate Lyase. Citrate lyase (300 $\mu \mathrm{L}, 40 \mathrm{mg} \mathrm{mL}^{-1}$ in PBS $1 \times$ ) was added to $1 \mathrm{~mL}$ of each $\mathrm{Cu}-\mathrm{NP}$ sample. The mixture was kept under vigorous stirring for $90 \mathrm{~min}$ at room temperature. Once this step was completed, samples were purified by ultrafiltration using 10 $\mathrm{kDa}$ cutoff filters and resuspended in water.

4.4. Synthesis of IONP-RGD. The cycloRGD peptide was covalently attached to carboxyl groups in the citrate coating by EDC/sulfoNHS chemistry, yielding IONP-RGD. 1-Ethyl-3-(3-dimethylaminopropyl)carbodiimide hydrochloride (EDC, $12 \mathrm{mg}$ ) and $15 \mathrm{mg}$ of $\mathrm{N}$-hydroxysulfosuccinimide (NHS) were added to $5 \mathrm{~mL}$ of IONP. The mixture was stirred at room temperature for $30 \mathrm{~min}$ and posteriorly purified by ultrafiltration using $30 \mathrm{kDa}$ cutoff filters. Filtered nanoparticles were diluted in buffer 4-(2-hydroxyethyl)-1-piperazineethanesulfonic acid (HEPES) pH 8 to $1.8 \mathrm{~mL}$ and $1 \mathrm{mg}$ of cycloRGD was then added and mixture stirred at room temperature for 60 min. Once this step was completed, the sample was purified by ultrafiltration and resuspended in saline.

4.5. Synthesis of Cu-NP-RGD. CycloRGD was covalently attached to carboxyl groups in the citrate coating by EDC/ sulfoNHS chemistry, yielding Cu-NP-RGD. 1-Ethyl-3-(3dimethylaminopropyl)carbodiimide hydrochloride (EDC, 12 $\mathrm{mg}$ ) and $15 \mathrm{mg}$ of $\mathrm{N}$-hydroxysulfosuccinimide (NHS) were added to $5 \mathrm{~mL}$ of $\mathrm{Cu}-\mathrm{NP}$. The mixture was stirred at room temperature for $30 \mathrm{~min}$ and posteriorly purified by ultrafiltration using $30 \mathrm{kDa}$ cutoff filters. Filtered nanoparticles were diluted in buffer HEPES, $\mathrm{pH} 8$, to $1.8 \mathrm{~mL}$ and $1 \mathrm{mg}$ of cycloRGD was then added, and the mixture stirred at room temperature for $60 \mathrm{~min}$. Once this step was completed, the sample was purified by ultrafiltration and resuspended in saline.

4.6. IONP and Cu-NP Hydrodynamic Size and $\zeta$ Potential Measurements. Hydrodynamic size and $\zeta$ potential of samples were measured using dynamic light scattering (DLS). A Zetasizer Nano ZS (Malvern Instruments) was used to this end. This device is equipped with a $\mathrm{He}-\mathrm{Ne}$ laser operating at $633 \mathrm{~nm}$ and $4 \mathrm{~mW}$ and an avalanche photodiode detector.

4.7. MRI Relaxation Properties of IONP and Cu-NP. Relaxometric properties of the samples were assessed by measuring longitudinal and transverse relaxation times. Four concentrations of each nanoparticle sample were selected, and longitudinal and transversal relaxation times of each one were measured using a Bruker Minispec mq60 contrast agent analyzer at $1.5 \mathrm{~T}$ and $37^{\circ} \mathrm{C}$. The $r_{1}$ and $r_{2}$ values were plotted against the Fe concentration (0, 0.25, 0.5, 1 , and $2 \mathrm{mM})$.
4.8. In Vivo MRI Acquisition. MRI equipment used in this study was an Agilent/Varian scanner (Agilent, Santa Clara, CA) equipped with a DD2 console and an actively shielded 205/120 gradient insert coil with $130 \mathrm{mT} \mathrm{m}^{-1}$ maximum gradient strength, a TX/RX volume quadrature coil, and a ${ }^{1} \mathrm{H}$ circularly polarized transmit-receive volume coil of $35 \mathrm{~mm}$ inner diameter and $30 \mathrm{~mm}$ active length built by Neos Biotec (Pamplona, Spain). For angiography study, mice weighing $30 \mathrm{~g}$ were anesthetized with $2 \%$ isoflurane and oxygen before being placed on a thermoregulated $\left(38{ }^{\circ} \mathrm{C}\right)$ mouse bed. An ophthalmic gel was applied in the eyes to prevent retinal damage due to drying. The three-dimensional gradient echo with magnetization transfer contrast (MTC) prepulse MRA was performed with the following parameters: min TR, 12.64 $\mathrm{ms}$; min TE, $2.32 \mathrm{~ms}$; flip angle, $20^{\circ}$; two averages; acquisition matrix, $256 \times 192 \times 128$; MTC flip angle, $810^{\circ}$; duration, 6 $\mathrm{ms}$; and offset frequency, $2000 \mathrm{~Hz}$. For tumor MRI experiments, axial $T_{1}$-weighted spin echo images were acquired with the following parameters: TR, $2072 \mathrm{~ms}$; TE, $27.9 \mathrm{~ms}$; NA, 8 ; matrix, $256 \times 256$; FOV, $30 \times 30 \mathrm{~mm}^{2}$; 20 consecutive 0.5 mm slices; and spectral width, $125000 \mathrm{~Hz}$.

\section{ASSOCIATED CONTENT}

\section{Supporting Information}

The Supporting Information is available free of charge on the ACS Publications website at DOI: 10.1021/acsomega.8b03004.

Mice tumor allograft model; physicochemical data of prepared nanoparticles; STEM-HAADF images of Cu1.7-NP, Cu4-NP, and Cu28-NP; TGA curves for Cu1.7-NP, Cu4-NP, Cu28-NP, and IONP; $T_{1}$ and $T_{2}$ values measured by relaxometry; $Z$-average and $\zeta$ potential values; and percentage of increase in MRI signal intensity (PDF)

\section{AUTHOR INFORMATION}

\section{Corresponding Author}

*E-mail: fherranz@iqm.csic.es.

ORCID $\odot$

Lucía Gutiérrez: 0000-0003-2366-3598

Sabino Veintemillas-Verdaguer: 0000-0002-3015-1470

María del Puerto Morales: 0000-0002-7290-7029

Fernando Herranz: 0000-0002-3743-0050

\section{Author Contributions}

The manuscript was written through contributions of all authors. All authors have given approval to the final version of the manuscript.

Notes

The authors declare no competing financial interest.

\section{ACKNOWLEDGMENTS}

This study was supported by grants from the Spanish Ministry for Economy and Competitiveness (MEyC) (SAF2016-79593P, MAT2017-88148-R, and SAF2017-84494-C2-R), Comunidad de Madrid (S2017/BMD-3875), and Instituto de Salud Carlos III (DTS16/00059). L.G. received financial support from the Ramon y Cajal subprogram (RYC-2014-15512). J.R.C. acknowledges funding from the Programa Red Guipuzcoana de Ciencia, Tecnología e Información (2018-CIEN-00005801). I.F.-B. thanks Comunidad de Madrid (B2017/BMD3875). This work was performed under the Maria de Maeztu 
Units of Excellence Program from the Spanish State Research Agency (Grant No. MDM-2017-0720).

\section{REFERENCES}

(1) Shen, Z.; Wu, A.; Chen, X. Iron Oxide Nanoparticle Based Contrast Agents for Magnetic Resonance Imaging. Mol. Pharm. 2017, 14, 1352-1364.

(2) Blanco-Andujar, C.; Walter, A.; Cotin, G.; Bordeianu, C.; Mertz, D.; Felder-Flesch, D.; Begin-Colin, S. Design of iron oxide-based nanoparticles for MRI and magnetic hyperthermia. Nanomedicine 2016, 11, 1889-1910.

(3) Pellico, J.; Ruiz-Cabello, J.; Fernández-Barahona, I.; Gutiérrez, L.; Lechuga-Vieco, A. V.; Enríquez, J. A.; Morales, M. P.; Herranz, F. One-Step Fast Synthesis of Nanoparticles for MRI: Coating Chemistry as the Key Variable Determining Positive or Negative Contrast. Langmuir 2017, 33, 10239-10247.

(4) Fantechi, E.; Campo, G.; Carta, D.; Corrias, A.; de Julián Fernández, C.; Gatteschi, D.; Innocenti, C.; Pineider, F.; Rugi, F.; Sangregorio, C. Exploring the Effect of Co Doping in Fine Maghemite Nanoparticles. J. Phys. Chem. C 2012, 116, 8261-8270.

(5) Moise, S.; Céspedes, E.; Soukup, D.; Byrne, J. M.; El Haj, A. J.; Telling, N. D. The cellular magnetic response and biocompatibility of biogenic zinc- and cobalt-doped magnetite nanoparticles. Sci. Rep. 2017, 7, No. 39922.

(6) Jang, J. T.; Nah, H.; Lee, J. H.; Moon, S. H.; Kim, M. G.; Cheon, J. Critical enhancements of MRI contrast and hyperthermic effects by dopant-controlled magnetic nanoparticles. Angew. Chem., Int. Ed. 2009, 48, 1234-1238.

(7) Yin, X.; Russek, S. E.; Zabow, G.; Sun, F.; Mohapatra, J.; Keenan, K. E.; Boss, M. A.; Zeng, H.; Liu, J. P.; Viert, A.; Liou, S.-H.; Moreland, J. Large T1 contrast enhancement using superparamagnetic nanoparticles in ultra-low field MRI. Sci. Rep. 2018, 8, No. 11863.

(8) Li, Z.; Wang, S. X.; Sun, Q.; Zhao, H. L.; Lei, H.; Lan, M. B.; Cheng, Z. X.; Wang, X. L.; Dou, S. X.; Max Lu, G. Q. Ultrasmall Manganese Ferrite Nanoparticles as Positive Contrast Agent for Magnetic Resonance Imaging. Adv. Healthcare Mater. 2013, 2, 958964.

(9) Zhang, M.; Cao, Y.; Wang, L.; Ma, Y.; Tu, X.; Zhang, Z. Manganese Doped Iron Oxide Theranostic Nanoparticles for Combined T 1 Magnetic Resonance Imaging and Photothermal Therapy. ACS Appl. Mater. Interfaces 2015, 7, 4650-4658.

(10) Xiao, N.; Gu, W.; Wang, H.; Deng, Y.; Shi, X.; Ye, L. T1-T2 dual-modal MRI of brain gliomas using PEGylated Gd-doped iron oxide nanoparticles. J. Colloid Interface Sci. 2014, 417, 159-165.

(11) Kang, H. Y.; Wang, H. P. Preparation of magnetic recoverable nanosize $\mathrm{Cu}-\mathrm{Fe} 2 \mathrm{O} 3 / \mathrm{Fe}$ photocatalysts. Environ. Sci. Technol. 2013, 47, 7380-7387.

(12) Thi, T. M.; Trang, N. T. H.; Van Anh, N. T. Effects of Mn, Cu doping concentration to the properties of magnetic nanoparticles and arsenic adsorption capacity in wastewater. Appl. Surf. Sci. 2015, 340, $166-172$.

(13) Sun, P.; Wang, C.; Zhou, X.; Cheng, P.; Shimanoe, K.; Lu, G.; Yamazoe, N. Cu-doped $\alpha$-Fe2O3hierarchical microcubes: Synthesis and gas sensing properties. Sens. Actuators, B 2014, 193, 616-622.

(14) Batoo, K. M.; Salah, D.; Kumar, G.; Kumar, A.; Singh, M.; Abd El-Sadek, M.; Mir, F. A.; Imran, A.; Jameel, D. A. Hyperfine interaction and tuning of magnetic anisotropy of $\mathrm{Cu}$ doped $\mathrm{CoFe} 2 \mathrm{O} 4$ ferrite nanoparticles. J. Magn. Magn. Mater. 2016, 411, 91-97.

(15) Zhou, S.-M.; Lou, S.-Y.; Wang, Y.-Q.; Chen, X.-L.; Liu, L.-S.; Yuan, H.-L. Preparation of $\mathrm{Cu}$-doped $\gamma$ - $\mathrm{Fe} 2 \mathrm{O} 3$ nanowires with high coercivity by chemical vapor deposition. J. Mater. Res. 2011, 26, 1634-1638.

(16) Lassoued, A.; Lassoued, M. S.; Dkhil, B.; Gadri, A.; Ammar, S. Structural, optical and morphological characterization of Cu-doped $\alpha$ $\mathrm{Fe} 2 \mathrm{O} 3$ nanoparticles synthesized through co-precipitation technique. J. Mol. Struct. 2017, 1148, 276-281.

(17) Abbasi Pour, S.; Shaterian, H. R.; Afradi, M.; Yazdani-ElahAbadi, A. Carboxymethyl cellulose (CMC)-loaded $\mathrm{Co}-\mathrm{Cu}$ doped manganese ferrite nanorods as a new dual-modal simultaneous contrast agent for magnetic resonance imaging and nanocarrier for drug delivery system. J. Magn. Magn. Mater. 2017, 438, 85-94.

(18) Wong, R. M.; Gilbert, D. A.; Liu, K.; Louie, A. Y. Rapid sizecontrolled synthesis of dextran-coated, $64 \mathrm{Cu}$-doped iron oxide nanoparticles. ACS Nano 2012, 6, 3461-3467.

(19) Pellico, J.; Ruiz-Cabello, J.; Saiz-Alía, M.; del Rosario, G.; Caja, S.; Montoya, M.; Fernández de Manuel, L.; Morales, M. P.; Gutiérrez, L.; Galiana, B.; Enríquez, J. A.; Herranz, F. Fast synthesis and bioconjugation of $68 \mathrm{Ga}$ core-doped extremely small iron oxide nanoparticles for PET/MR imaging. Contrast Media Mol. Imaging 2016, 11, 203-210.

(20) Li, Z.; Yi, P. W.; Sun, Q.; Lei, H.; Li Zhao, H.; Zhu, Z. H.; Smith, S. C.; Lan, M. B.; Lu, G. Q. M. Ultrasmall Water-Soluble and Biocompatible Magnetic Iron Oxide Nanoparticles as Positive and Negative Dual Contrast Agents. Adv. Funct. Mater. 2012, 22, 23872393.

(21) Hu, F.; Jia, Q.; Li, Y.; Gao, M. Facile synthesis of ultrasmall PEGylated iron oxide nanoparticles for dual-contrast $T_{1^{-}}$and $T_{2^{-}}$ weighted magnetic resonance imaging. Nanotechnology 2011, 22, No. 245604.

(22) Tromsdorf, U. I.; Bruns, O. T.; Salmen, S. C.; Beisiegel, U.; Weller, H. A Highly Effective, Nontoxic T 1 MR Contrast Agent Based on Ultrasmall PEGylated Iron Oxide Nanoparticles. Nano Lett. 2009, 9, 4434-4440.

(23) Zhou, Z.; Wang, L.; Chi, X.; Bao, J.; Yang, L.; Zhao, W.; Chen, Z.; Wang, X.; Chen, X.; Gao, J. Engineered Iron-Oxide-Based Nanoparticles as Enhanced T1 Contrast Agents for Efficient Tumor Imaging. ACS Nano 2013, 7, 3287-3296.

(24) Taboada, E.; Rodríguez, E.; Roig, A.; Oró, J.; Roch, A.; Muller, R. N. Relaxometric and magnetic characterization of ultrasmall iron oxide nanoparticles with high magnetization. Evaluation as potential T1 magnetic resonance imaging contrast agents for molecular imaging. Langmuir 2007, 23, 4583-4588.

(25) Kim, B. H.; Lee, N.; Kim, H.; An, K.; Park, Y.; Il Choi, Y.; Shin, K.; Lee, Y.; Kwon, S. G.; Na, H.; Bin Park, J.-G.; Ahn, T.-Y.; Kim, Y.W.; Moon, W. K.; Choi, S. H.; Hyeon, T. Large-Scale Synthesis of Uniform and Extremely Small-Sized Iron Oxide Nanoparticles for High-Resolution T1 Magnetic Resonance Imaging Contrast Agents. J. Am. Chem. Soc. 2011, 133, 12624-12631.

(26) Park, J. C.; Lee, G. T.; Kim, H.; Sung, B.; Lee, Y.; Kim, M.; Chang, Y.; Seo, J. H. Surface Design of Eu-Doped Iron Oxide Nanoparticles for Tuning the Magnetic Relaxivity. ACS Appl. Mater. Interfaces 2018, 10, 25080-25089.

(27) Saraswathy, A.; Nazeer, S. S.; Jeevan, M.; Nimi, N.; Arumugam, S.; Harikrishnan, V. S.; Varma, P. R. H.; Jayasree, R. S. Citrate coated iron oxide nanoparticles with enhanced relaxivity for in vivo magnetic resonance imaging of liver fibrosis. Colloids Surf., B 2014, 117, 216224.

(28) Shen, T.; Weissleder, R.; Papisov, M.; Bogdanov, A.; Brady, T. J. Monocrystalline iron oxide nanocompounds (MION): Physicochemical properties. Magn. Reson. Med. 1993, 29, 599-604.

(29) Yang, H.; Zhuang, Y.; Sun, Y.; Dai, A.; Shi, X.; Wu, D.; Li, F.; $\mathrm{Hu}, \mathrm{H}$.; Yang, S. Targeted dual-contrast T1- and T2-weighted magnetic resonance imaging of tumors using multifunctional gadolinium-labeled superparamagnetic iron oxide nanoparticles. Biomaterials 2011, 32, 4584-4593.

(30) Shen, Z.; Song, J.; Zhou, Z.; Yung, B. C.; Aronova, M. A.; Li, Y.; Dai, Y.; Fan, W.; Liu, Y.; Li, Z.; Ruan, H.; Leapman, R. D.; Lin, L.; Niu, G.; Chen, X.; Wu, A. Dotted Core-Shell Nanoparticles for $T_{1}$ Weighted MRI of Tumors. Adv. Mater. 2018, 30, No. 1803163. 